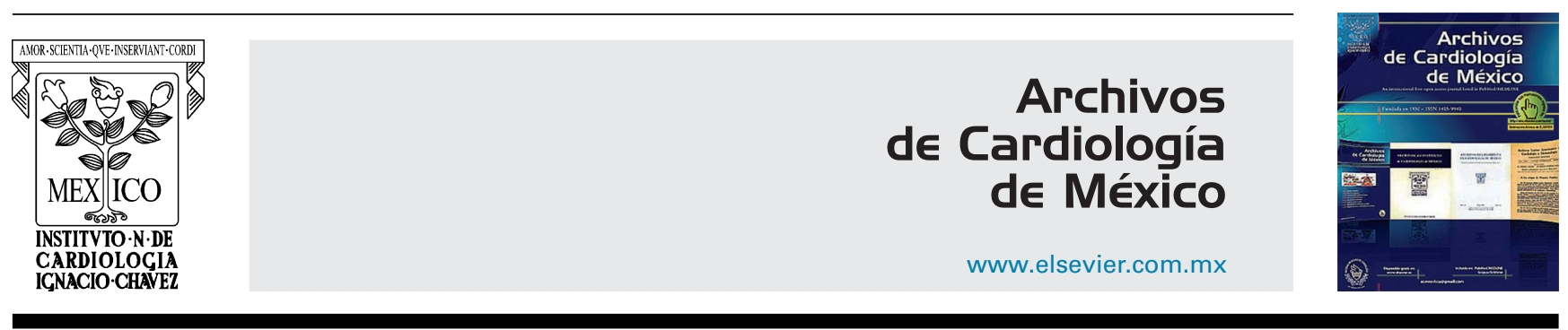

INVESTIGACIÓN CLÍNICA

\title{
Evaluación de la estrategia de terapia antitrombótica posterior al cambio valvular aórtico con válvulas biológicas en pacientes de bajo riesgo trombótico
}

\author{
Eduardo Aceves-Velázquez ${ }^{\mathrm{a}, *}$, Gerardo Vieyra-Herrera ${ }^{\mathrm{b}}$, Laura Rodríguez-Chávez ${ }^{\mathrm{b}}$ \\ y Valentín Herrera-Alarcón ${ }^{c}$
}

\author{
a Cardiología, Instituto Nacional de Cardiología Ignacio Chávez, Ciudad de México, México \\ b Departamento de Consulta Externa, Instituto Nacional de Cardiología Ignacio Chávez, Ciudad de México, México \\ c Departamento de Cirugía, Instituto Nacional de Cardiología Ignacio Chávez, Ciudad de México, México
}

Recibido el 22 de febrero de 2017; aceptado el 19 de junio de 2017

\author{
PALABRAS CLAVE \\ Cambio valvular \\ aórtico; \\ Prótesis biológica; \\ Anticoagulación; \\ Trombosis valvular; \\ Antagonista de \\ vitamina $\mathrm{K}$; \\ Aspirina; \\ México
}

\begin{abstract}
Resumen De acuerdo a las guías actuales, aún es materia de debate el uso de anticoagulación en los primeros 3 meses en pacientes de bajo riesgo trombótico sometidos a cambio valvular aórtico con prótesis biológicas. En base a la evidencia actual, la aspirina a dosis bajas es razonable como alternativa a los antagonistas de la vitamina K (AVK) durante el posquirúrgico temprano en pacientes con prótesis biológicas en posición aórtica.

Se comparó la incidencia de complicaciones trombóticas o hemorrágicas de acuerdo a la estrategia de terapia antitrombótica en los pacientes de bajo riesgo trombótico sometidos a cambio valvular aórtico con válvulas biológicas en el Instituto Nacional de Cardiología Ignacio Chávez. La hipótesis: la aspirina como monoterapia antitrombótica tiene un efecto benéfico comparado con los AVK.

Se estudiaron los pacientes de bajo riesgo trombótico sometidos a cambio valvular aórtico con válvulas biológicas en el Instituto Nacional de Cardiología Ignacio Chávez. Se incluyeron los pacientes operados del año 2011 al 2015. Se identificó en el seguimiento a un año la presencia de complicaciones trombóticas o hemorrágicas y si se manejaron con cualquiera de las siguientes: aspirina únicamente, AVK solo y la combinación aspirina más AVK.

Se analizaron 231 pacientes. Solo se presentó una complicación hemorrágica en un paciente tratado con AVK. No hubo complicaciones trombóticas.

No se presentaron complicaciones trombóticas en pacientes que no recibieron anticoagulación oral formal durante los primeros 3 meses posquirúrgicos, lo que indica que es seguro el uso de aspirina como monoterapia en estos pacientes de bajo riesgo trombótico.

(c) 2017 Instituto Nacional de Cardiología Ignacio Chávez. Publicado por Masson Doyma México S.A. Este es un artículo Open Access bajo la licencia CC BY-NC-ND (http://creativecommons. org/licenses/by-nc-nd/4.0/).
\end{abstract}

\footnotetext{
* Autor para correspondencia. Prof. Manuel Acosta 1. Int. 101. Delegación La Magdalena Contreras. Ciudad de México. CP 10100. Teléfono: 0445566127326.

Correo electrónico: laloaceves@gmail.com (E. Aceves-Velázquez).
} 


\section{KEYWORDS}

Aortic valve

replacement;

Bioprosthesis;

Anticoagulation;

Valvular thrombosis;

Vitamina $\mathrm{K}$

antagonist;

Aspirin;

Mexico
Evaluation of the antithrombotic strategy in low thrombotic risk patients who underwent aortic valve replacement with a bioprosthesis

Abstract According to current guidelines, in patients without additional risk factors who have undergone aortic valve replacement with a bioprosthesis, anticoagulation in the first 3 months after surgery is still a matter of debate. According to current evidence, aspirin in low doses is a reasonable alternative to vitamin $\mathrm{K}$ antagonists (VKA).

A comparison is made between the incidence of thrombotic and haemorrhagic complications in patients with low thrombotic risk who underwent aortic valve replacement with a bioprosthesis in the National Institute of Cardiology of Ignacio Chávez of Mexico. The hypothesis: aspirin as monotherapy has a beneficial effect compared to VKA.

The studied patients were the low thrombotic risk patients who underwent aortic valve replacement with a bioprosthesis in the National Institute of Cardiology of Ignacio Chávez of Mexico from 2011 to 2015. The groups studied were: aspirin only, VKA only, and the combination of VKA plus aspirin. The patients were retrospectively followed-up for 12 months, and the thrombotic and haemorrhagic complications were documented.

Of the 231 patients included in the study, only one patient in the VKA only group presented with a haemorrhagic complication. No thrombotic complications were observed.

In the present study no thrombotic complications were observed in patients who did not receive anticoagulation in the first 3 months after an aortic valve replacement with a bioprosthesis after a follow up period of 12 months. This suggests that the use of aspirin only is safe during this period.

(C) 2017 Instituto Nacional de Cardiología Ignacio Chávez. Published by Masson Doyma México S.A. This is an open access article under the CC BY-NC-ND license (http://creativecommons. org/licenses/by-nc-nd/4.0/).

\section{Introducción}

Las prótesis valvulares biológicas son menos trombogénicas que las prótesis mecánicas y no requieren anticoagulación a largo plazo. La mayoría de las prótesis biológicas son de origen porcino. Las válvulas porcinas y de pericardio bovino tienen una trombogenicidad similar.

Actualmente los antagonistas de la vitamina K (AVK) son los únicos anticoagulantes aprobados para los pacientes sometidos a cambios valvulares. De estos, la warfarina tiene una vida media de $40 \mathrm{~h}$, el acenocumarol de entre 8 y $11 \mathrm{~h}$, la fluindiona de $30 \mathrm{~h}$ y el fenoprocumon de 3 a 5 días ${ }^{1}$. El international normalised ratio (INR) es el único método estándar para reportar la intensidad de anticoagulación con estos medicamentos. El uso de estos medicamentos significa además el riesgo de sangrado (siendo incluso mayor en pacientes de edad avanzada, en quienes más se implantan válvulas biológicas) además del costo económico de su control (toma de muestra de laboratorio para INR y consulta médica para su ajuste).

La mayor ventaja que ofrecen las prótesis biológicas en posición aórtica es que no cuentan con la necesidad de anticoagulación de por vida comparadas con las prótesis mecánicas.

El riesgo de eventos tromboembólicos en pacientes con una válvula mecánica aórtica es del $1-2 \%$ contra $0.7 \%$ con una válvula biológica, incluso con terapia antitrombótica adecuada $^{2}$. En pacientes con válvulas biológicas aórticas la terapia antitrombótica es obligada ante la presencia de factores de riesgo tromboembólicos como la fibrilación auricular, un tromboembolismo previo, disfunción ventricular izquierda y los diferentes estados hipercoagulables. En pacientes sin estos factores de riesgo, el régimen antitrombótico postoperatorio es aún materia de debate. En base a la evidencia actual, un régimen de aspirina a dosis bajas es razonable como alternativa a los AVK durante el posquirúrgico temprano ( 3 meses) en pacientes con prótesis biológicas en posición aórtica. A pesar de esto, por la percepción de incremento en el riesgo tromboembólico en los primeros 3 meses postoperatorios en pacientes con válvulas protésicas biológicas, la mayoría de los estudios han tratado a los pacientes con AVK durante los primeros 3 meses. Debido a que sí se ha comprobado que el riesgo de tromboembolismo es bajo posterior a estos 3 meses, el riesgo de sangrado supera cualquier beneficio de la terapia con AVK si esta se continúa después de 3 meses. Independientemente de la elección como terapia inicial, todos los pacientes deben recibir dosis bajas de aspirina ( $\leq 100 \mathrm{mg}$ al día) tras los primeros 3 meses.

En la tabla 1 se exponen las recomendaciones actuales de las diferentes sociedades cardiovasculares ${ }^{3-7}$. En la figura 1 se muestra el algoritmo de la terapia antitrombótica para pacientes con válvulas protésicas biológicas.

La práctica médica para la prevención de eventos trombóticos tempranos posterior a un cambio valvular aórtico biológico varía de manera importante entre centros e incluso dentro de los mismos. A pesar de las recomendaciones de las diferentes sociedades continúa habiendo diferencias importantes de opinión en la manera de proceder en el manejo médico de la terapia antitrombótica. En una encuesta, Vaughan y Waterworth encontraron que solo el $16 \%$ de los médicos consultados se adhirieron a las recomendaciones 
Tabla 1 Recomendaciones de guías actuales

\begin{tabular}{|c|c|c|}
\hline Organización, año & Recomendación & Evidencia \\
\hline ESC, 2012 & $\begin{array}{l}\text { Aspirina a dosis bajas debe ser considerada en los primeros } 3 \text { meses } \\
\text { posteriores a la implantación de una prótesis biológica en posición aórtica }\end{array}$ & $2 C$ \\
\hline ACC/AHA, 2014 & $\begin{array}{l}\text { Aspirina } 75-100 \mathrm{mg} \text { al día es razonable en todos los pacientes con una } \\
\text { prótesis biológica en posición aórtica. Anticoagulación con un antagonista } \\
\text { de vitamina K para mantener un INR de } 2.5 \text { puede ser también razonable } \\
\text { durante los primeros } 3 \text { meses posteriores al cambio valvular }\end{array}$ & $2 B$ \\
\hline ACCP, 2012 & $\begin{array}{l}\text { En pacientes con una prótesis biológica en posición aórtica, se aconseja } \\
\text { aspirina ( } 50-100 \mathrm{mg}) \text { además de la terapia con antagonistas de la vitamina } \\
\mathrm{K} \text { durante los primeros } 3 \text { meses }\end{array}$ & $2 C$ \\
\hline EACTS, 2008 & $\begin{array}{l}\text { Después del cambio valvular aórtico, la terapia anticoagulante es } \\
\text { razonablemente segura y puede ser benéfica. La terapia antiplaquetaria } \\
\text { únicamente es una alternativa aceptable }\end{array}$ & $2 B$ \\
\hline CCS, 2004 & $\begin{array}{l}\text { Se recomienda antagonista de vitamina } \mathrm{K} \text { durante los } 3 \text { meses posteriores } \\
\text { al cambio valvular aórtico con prótesis biológica }\end{array}$ & $2 C$ \\
\hline BSH, 1998 & $\begin{array}{l}\text { No se recomienda el uso de anticoagulantes orales en postoperados de } \\
\text { cambio valvular aórtico con prótesis biológica en pacientes con ritmo } \\
\text { sinusal }\end{array}$ & 1B \\
\hline
\end{tabular}

de la ACCP/AHA en cuanto a la anticoagulación de este tipo de pacientes. La mayoría de los médicos (52\%) nunca utilizaron AVK en el postoperatorio, y de esos, el 78\% iniciaron ASA en su lugar. El otro 22\% no utilizó AVK ni ASA ${ }^{8}$. En 2007, Colli et al. publicaron una revisión al respecto y encontraron que, en EE. UU., el $60 \%$ de los cirujanos cardiotorácicos creen que la terapia antiplaquetaria es preferible a la anticoagulación para pacientes con comorbilidades mayores. En una encuesta propia de esos autores, reportaron que el $25 \%$ de los centros médicos europeos administran únicamente ASA en el postoperatorio temprano, sin anticoagulantes orales ${ }^{9}$.

Múltiples estudios, la mayoría de ellos retrospectivos y observacionales, han comparado la incidencia de eventos trombóticos y complicaciones hemorrágicas entre el

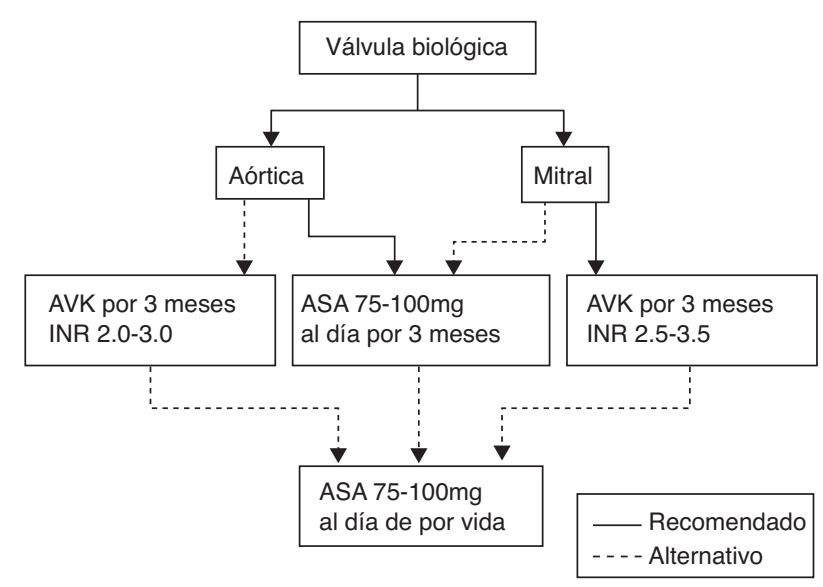

Figura 1 Algoritmo para la terapia antitrombótica para prótesis valvulares biológicas basadas en las recomendaciones de la ACC/AHA 2014. ACC: American College of Cardiology; AHA: American Heart Association. tratamiento a base de antiagregación plaquetaria y el de anticoagulación formal con y sin antiagregante plaquetario.

Durante la revisión bibliográfica no se encontraron estudios enfocados a pacientes latinoamericanos a este respecto.

Los grupos más grandes reportados a los cuales no se les administró terapia antitrombótica durante los primeros 3 meses postoperatorios consistieron en 156 y 136 pacientes. La tasa de tromboembolismo a un año fue del $1.3 \%$ en válvulas aórticas y a 7 años del 1.5 y $1.7 \%$ por años/paciente para válvulas aórticas y mitrales respectivamente ${ }^{10}$.

En 1988, Turpie et al. no encontraron diferencias en los eventos embólicos mayores y reportaron una baja tasa de sangrado mayor con anticoagulación a dosis bajas con INR entre 2 y 2.3 durante los primeros 3 meses posteriores a la cirugía $^{11}$.

En 2 estudios observacionales pequeños en pacientes con válvulas biológicas (aórtica y mitral) que recibieron anticoagulación durante los primeros 3 meses posquirúrgicos seguidos de ninguna terapia antitrombótica reportaron una tasa de tromboembolismo del 1.5-2\% por año/paciente después de un seguimiento de 3-7 años ${ }^{12,13}$.

Heras et al. observaron una alta tasa de eventos tromboembólicos en pacientes que no recibieron anticoagulación, particularmente en pacientes con cambio valvular mitral durante los primeros 10 días postoperatorios. Concluyeron que se debe administrar anticoagulación oral para mantener un INR de 3-4.5 durante por lo menos 3 meses, sin embargo, no hicieron distinción entre prótesis en posición mitral o aórtica además de que a pesar de que a un tercio de los pacientes se les dio anticoagulación, dos tercios se encontraban en rangos subterapéuticos de INR durante estos primeros 10 días posquirúrgicos ${ }^{14}$.

En 1995, Orszulak et al. encontraron que el uso temprano de warfarina posterior al cambio valvular aórtico con prótesis biológica era beneficioso solo en pacientes 
con características de alto riesgo como función ventricular deprimida, edad mayor y fibrilación auricular o uso de marcapasos preoperatorios ${ }^{15}$.

Moinuddeen et al. estudiaron a pacientes con cambio valvular aórtico con prótesis biológica entre 1987 y 1996. Un grupo fue tratado con warfarina por 3 meses y el otro no recibió anticoagulación postoperatoria. No encontraron diferencias estadísticamente significativas para eventos isquémicos o hemorrágicos entre ambos grupos por lo que concluyeron que la anticoagulación posterior a la cirugía no es necesaria ${ }^{16}$.

En 1998, Tiede et al., en un artículo de revisión recomendaron la anticoagulación con warfarina en todos los pacientes $^{17}$.

Babin-Ebell et al. y Joyce y Nelson no encontraron beneficio con warfarina comparado con ASA. Además, en 378 pacientes que fueron sometidos a cambio valvular aórtico tratados con ASA, warfarina o ninguna de las 2. Blair et al. encontraron una media del $0.8 \pm 0.2 \%$ por años/paciente de incidencia de embolismos asociados con ASA, menor del $2.9 \pm 1.6 \%$ con warfarina y $1.5 \pm 0.6 \%$ sin tratamiento. Los autores concluyeron su artículo con la recomendación del uso de ASA únicamente posterior al cambio valvular $^{18-20}$.

En 2004, Gherli et al. reportaron que no hubo diferencia en eventos isquémicos cerebrales, hemorragia mayor o supervivencia en 141 pacientes que recibieron warfarina por 3 meses comparados con 108 pacientes tratados con aspirina únicamente ${ }^{21}$.

Mistiaen et al. analizaron a 500 pacientes mayores que recibieron prótesis Carpentier-Edwards y encontraron en el análisis multivariado que el uso de AVK incluso aumenta el riesgo de tromboembolismo con una razón de riesgo de 3 con un seguimiento a 4 años $^{22}$.

En un análisis retrospectivo de 1,151 pacientes que fueron sometidos a un cambio valvular aórtico en la Clínica Mayo encontraron que los accidentes cerebrovasculares ocurrieron en el $2.4 \%$ de los pacientes anticoagulados y en el $1.9 \%$ de los no anticoagulados, una diferencia estadísticamente no significativa. Los autores concluyeron que la anticoagulación con warfarina en el postoperatorio temprano no protege contra eventos neurológicos ${ }^{17}$.

En el estudio TRAC, un estudio multicéntrico aleatorizado, se comparó el agente antiplaquetario triflusal con acenocumarol en la prevención de tromboembolismo posterior a cambio valvular aórtico y mitral, y encontraron que el agente era tan seguro y efectivo como el AVK en estos pacientes $^{23}$.

En 2005 Colli et al. presentaron los resultados del estudio WoA Epic que comparó la warfarina contra ASA posterior al cambio valvular aórtico con la prótesis St. Jude Epic en pacientes sin otro factor de riesgo tromboembólico. Las tasas de sangrado mayor, supervivencia libre de eventos vasculares cerebrales y supervivencia en general no fueron significativamente diferentes en ambos grupos. Concluyeron que no hay evidencia para apoyar la anticoagulación con warfarina para prevenir la trombosis valvular o tromboembolismo arterial en los pacientes con prótesis St. Jude Epic $^{24}$.

Jamieson et al. presentaron un estudio retrospectivo de 1,372 pacientes que fueron sometidos a cambio valvular $y$ aunque limitaron su definición de eventos tempranos a los primeros 30 días únicamente, el análisis univariado mostró que la terapia antiplaquetaria fue el único tratamiento protector contra eventos tromboembólicos mayores ${ }^{25}$.

Estas observaciones orientan a que en pacientes sin factores de riesgo tromboembólico es suficiente la terapia antiplaquetaria como protección antitrombótica durante el postoperatorio temprano en pacientes con cambio valvular aórtico con prótesis biológicas ${ }^{19,20,26}$, sin embargo, hasta la fecha no se ha probado de forma definitiva. Actualmente todos los manufacturadores de prótesis biológicas recomiendan el seguimiento de las guías establecidas así que ninguna válvula esta recomendada con régimen antiplaquetario únicamente.

\section{Métodos}

Se trata de un estudio de tipo retrospectivo. La población estudiada fueron los pacientes de bajo riesgo trombótico sometidos a cambio valvular aórtico con válvulas biológicas en el Instituto Nacional de Cardiología Ignacio Chávez. La muestra consistió en los pacientes con estas características en el periodo de 2011 a 2014.

Los criterios de inclusión fueron hombres y mujeres, mayores de 18 años, admitidos en el Instituto Nacional de Cardiología Ignacio Chávez y pacientes operados de cambio valvular aórtico por prótesis biológica durante el periodo en estudio. Los criterios de exclusión fueron pacientes con indicación para terapia anticoagulante independiente al cambio valvular pórtico con prótesis biológica, fibrilación auricular, tromboembolismo previo, estados hipercoagulables, expedientes con falta de datos necesarios para su análisis estadístico, pacientes sin seguimiento a un año por lo menos y pacientes que fallecieron antes de su egreso hospitalario.

El análisis de los resultados se llevó a cabo con métodos y modelos de análisis de los datos según tipo de variables: medidas de frecuencia, medidas de dispersión y porcentajes. Para esto se utilizaron los programas: Microsoft Excel for Mac v 15.24, Microsoft 2016 y SPSS Statistics $\vee 24.0$, IBM 2016.

\section{Resultados}

Se encontraron 231 pacientes operados de cambio valvular aórtico con prótesis biológica en el periodo de estudio (2011-2014). De estos pacientes, 38 fueron excluidos por no cumplir con el seguimiento mínimo requerido de un año, 27 por haber fallecido intrahospitalariamente, 18 pacientes tuvieron una indicación extra para anticoagulación formal y un paciente fue llevado a cambio por válvula mecánica al mes de su cambio valvular inicial. Finalmente se hizo el análisis con 147 pacientes, de los cuales, 61 recibieron únicamente un AVK (durante por lo menos 3 meses), 68 solo aspirina, 17 recibieron un AVK y aspirina, y un paciente no recibió ninguna de las 2 (fig. 2 ).

Por año se estudiaron los siguientes: en 2011, 34 casos; en 2012, 43; en 2013, 40 y en 2014, 30 (fig. 3). El promedio de edad de todos los pacientes incluidos fue de 59.7 (desviación estándar: 13.4), 92 hombres y 55 mujeres que representan el 63 y el 37\% respectivamente. La causa más frecuente de cirugía fue la estenosis aórtica con un total de 105 casos 
231 pacientes operados de cambio valvular aórtico

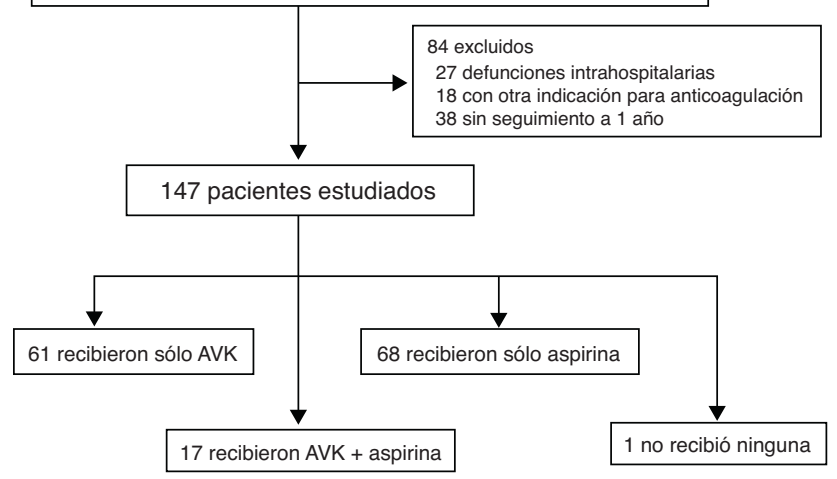

Figura 2 Diagrama de flujo para pacientes con cambio valvular aórtico por prótesis biológica. AVK: antagonista de la vitamina $\mathrm{K}$.

Casos estudiados por año

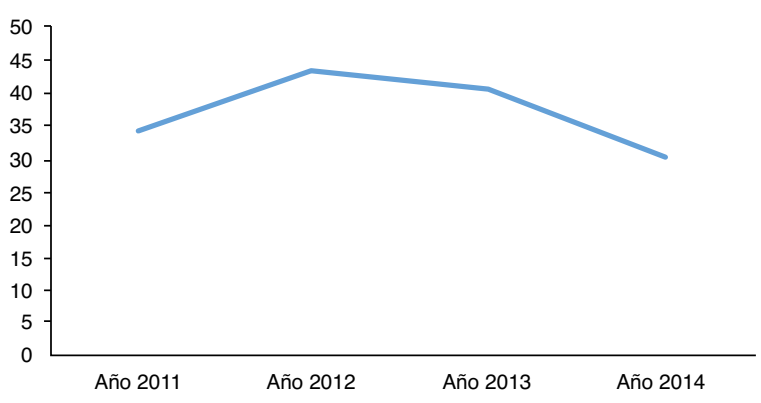

Figura 3 Casos estudiados por año.

(71\%), la insuficiencia aórtica fue la causa en 24 ocasiones (16\%) y la enfermedad mixta (estenosis e insuficiencia), en 18 (12\%) (fig. 4). La válvula más frecuentemente utilizada fue la INC en 127 ocasiones (89\%) seguida de la CarpentierEdwards 17 veces (11\%) y por último la St. Jude Epic en 3 (2\%)

\section{Tipo de enfermedad aórtica}

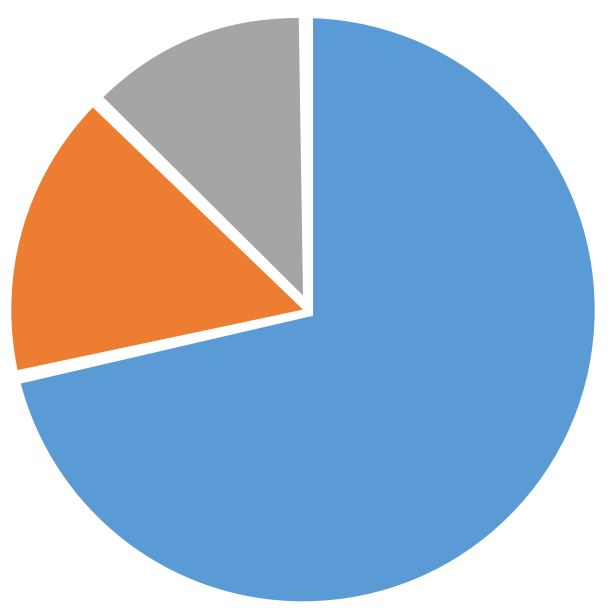

Estenosis Insuficiencia Mixta

Figura 4 Tipo de enfermedad aórtica.
Tipos de válvulas utilizadas

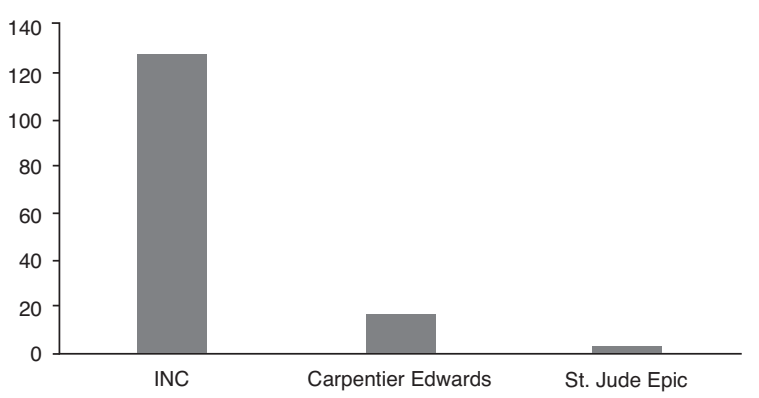

Figura 5 Tipos de válvulas utilizadas. INC: Instituto Nacional de Cardiología.

(fig. 5). En cuanto al tamaño de válvulas, la de menor tamaño fue la $18 \mathrm{~mm}$, la mayor fue de $30 \mathrm{~mm}$ con una mediana de $22 \mathrm{~mm}$ y moda también de $22 \mathrm{~mm}$.

En la tabla 2 se representan las características demográficas de los pacientes.

Como se observa en la tabla, en las únicas variables donde se encontraron diferencias significativas fueron el EuroSCORE 2 que fue mayor en los pacientes que solo recibieron aspirina, en IAM previo, dislipidemia, hipertensión arterial sistémica, diabetes y otras valvulopatías concomitantes que fueron antecedentes más prevalentes en los pacientes que terminaron recibiendo ambas, AVK y aspirina. Hay que reconocer también que los pacientes que terminaron recibiendo terapia doble ya tomaban aspirina previamente.

Todos los pacientes que recibieron aspirina $(n=85$ en ambos grupos, solo aspirina y AVK + aspirina) la recibieron durante todo el seguimiento de 12 meses a una dosis de $100 \mathrm{mg} / 24 \mathrm{~h}$. Los pacientes que solo recibieron AVK $(n=61)$ tuvieron una media de 7 meses en su prescripción con una desviación estándar de 4 y dentro del grupo que recibió ambas, una media de 5.8 con desviación estándar de 3.9 meses. La dosis recibida de vitamina $\mathrm{K}$ fue variable en cada paciente de acuerdo a su control en clínica de anticoagulantes (INR meta entre 2 y 3 ).

En cuanto al seguimiento de las guías de práctica clínica mencionadas anteriormente, solo 10 pacientes $(6 \%)$ fueron manejados como se recomienda en las guías de la ACC/AHA 2014 con aspirina de manera continua y AVK durante los primeros 3 meses posquirúrgicos. El $57 \%$ de los pacientes estudiados recibieron aspirina, ya sea como monoterapia o combinada con un AVK, todos los pacientes que recibieron aspirina la tomaron durante todo el año de seguimiento. El $41 \%$ recibieron únicamente un $\mathrm{AVK}$ con una media de $7 \mathrm{y}$ desviación estándar de 4 meses. El 32\% de estos pacientes recibieron el AVK durante por lo menos el año que les dimos seguimiento.

La mortalidad de los pacientes estudiados fue de 0.

Durante el periodo de estudio no se encontraron eventos de trombosis valvular en ningún paciente de ninguno de los 4 grupos estudiados. Un paciente dentro del grupo de solo AVK presentó un derrame pericárdico hemorrágico que lo llevó a taponamiento cardiaco y ventana pericárdica. Posterior a su egreso se reinició la anticoagulación oral y no presentó eventos hemorrágicos posteriores. 
Tabla 2 Características demográficas de la muestra

\begin{tabular}{|c|c|c|c|c|}
\hline Variable & $\begin{array}{l}\text { Solo AVK } \\
n=61\end{array}$ & $\begin{array}{l}\text { Solo aspirina } \\
\mathrm{n}=68\end{array}$ & $\begin{array}{l}\text { AVK + aspirina } \\
\mathrm{n}=17\end{array}$ & $\begin{array}{l}\text { Ninguna } \\
n=1\end{array}$ \\
\hline Edad, media (DE) & $57(13.3)$ & $61(14)$ & $59(9.3)$ & 83 \\
\hline Hombres, n (\%) & $40(65)$ & $41(60)$ & $10(58)$ & 1 \\
\hline EuroSCORE 2, media (DE) & $2.5(2.9)$ & $2.8(4.6)$ & $2.1(1.9)$ & 1.6 \\
\hline FEVI previa, media (DE) & $53(12.5)$ & $55(15)$ & $54(14)$ & 68 \\
\hline Cambio valvular previo, n (\%) & 0 & $1(1)$ & 0 & 0 \\
\hline Cirugía de revascularización previa, n (\%) & 0 & 0 & 0 & 0 \\
\hline Intervencionismo previo, n (\%) & 0 & $3(4)$ & 0 & 0 \\
\hline IAM previo, n (\%) & $1(1)$ & $3(4)$ & $2(11)$ & 0 \\
\hline Dislipidemia, n (\%) & $7(9)$ & 13 (19) & $4(23)$ & 0 \\
\hline Hipertensión arterial sistémica, n (\%) & $27(44)$ & $31(45)$ & $10(58)$ & 1 \\
\hline Diabetes mellitus, $\mathrm{n}(\%)$ & $8(13)$ & $22(32)$ & $6(35)$ & 0 \\
\hline Tabaquismo, n (\%) & $13(21)$ & $15(22)$ & $4(23)$ & 1 \\
\hline Uso previo de aspirina, n (\%) & $7(11)$ & $15(22)$ & $7(41)$ & 0 \\
\hline Uso previo de AVK, $\mathrm{n}(\%)$ & 0 & $1(1)$ & 0 & 0 \\
\hline Valvulopatía asociada, n (\%) & $15(24)$ & $7(10)$ & $2(11)$ & 0 \\
\hline Revascularización concomitante, n (\%) & $1(1)$ & $8(11)$ & $3(17)$ & 0 \\
\hline Reexploración quirúrgica, n (\%) & $8(13)$ & $12(17)$ & $2(11)$ & 0 \\
\hline
\end{tabular}

AVK: antagonistas de la vitamina K; DE: desviación estándar; IAM: infarto agudo de miocardio; FEVI: fracción de expulsión del ventrículo izquierdo.

\section{Discusión}

En la actualidad, la mayoría de las sociedades internacionales (ACC/AHA, ACCP, EACTS, CCS $)^{4-7}$ recomiendan el uso de AVK durante por lo menos los primeros 3 meses posquirúrgicos en pacientes sometidos a cambio valvular aórtico con prótesis biológica además de aspirina de por vida, independientemente de si son o no pacientes de bajo riesgo trombótico. Esto a pesar de que el nivel de evidencia es $2 \mathrm{~B}$ (ACC/AHA, EACTS) o $2 \mathrm{C}$ (ACCP, CCS). Las guías de la Sociedad Europea de Cardiología hacen la siguiente recomendación: «aspirina a dosis bajas debe ser considerada en los primeros 3 meses posteriores a la implantación de una prótesis biológica en posición aórtica» ${ }^{3}$. La guía de 1998 de la Sociedad Británica de Hematología es la única en pronunciarse en contra del uso de los anticoagulantes orales en estos pacientes de bajo riesgo trombótico ${ }^{6}$.

De acuerdo a nuestra experiencia institucional, se sabía que el porcentaje de adherencia a esta recomendación sería baja entre cardiólogos del Instituto Nacional de Cardiología Ignacio Chávez de la Ciudad de México. Como se mencionó anteriormente, en encuestas internacionales previas ${ }^{8,9}$, se encontró que un muy bajo porcentaje de médicos (16\%) seguían la recomendación de utilizar un AVK en el postoperatorio e incluso hasta el $22 \%$ no utilizaban terapia antitrombótica aguda. En este estudio encontramos que solo en el $10 \%$ de los casos se siguió la recomendación de la ACC/AHA de 2014 de anticoagular formalmente durante los primeros 3 meses además de aspirina de por vida, un número muy cercano a la información previamente presentada. Sorpresivamente, encontramos también que solo el $57 \%$ de los pacientes estudiados recibieron aspirina, ya sea como monoterapia o combinada con un AVK. Por otra parte, el $41 \%$ recibieron únicamente un AVK con una media de 7 y desviación estándar de 4 meses, incluso el $32 \%$ de estos pacientes recibieron el AVK durante por lo menos el año que les dimos seguimiento.

Como menciona la evidencia, un régimen de aspirina a dosis bajas es razonable como alternativa a los AVK durante el posquirúrgico temprano (3 meses) en pacientes con prótesis biológicas en posición aórtica ya que las tasas de trombosis no presentan diferencias significativas a favor de un grupo u otro. A favor de esta conclusión se encuentran múltiples estudios ${ }^{11,15,16,18,19,22,23,25}$. En este estudio, como se mencionó, no se encontraron casos de trombosis valvular en ninguno de los 4 grupos estudiados. Esto a pesar de que los pacientes que recibieron aspirina presentaban puntajes de EuroSCORE 2 más altos. Durante el estudio solo un paciente evidenció un evento hemorrágico, que fue en el grupo de pacientes que solo recibieron AVK.

Otra opción es la de no administrar tratamiento antitrombótico alguno, como ya fue estudiado por Blair en 1990 y Brueck 2007 donde obtuvieron una taza de trombosis al año de $1.3 \%$ en válvulas biológicas en posición aórtica $^{10,20}$. Debido a que en este estudio únicamente un paciente $(<1 \%)$ no recibió terapia antitrombótica alguna, no podemos obtener conclusiones al respecto. Cabe mencionar que no presentó trombosis de la válvula.

Las limitaciones que encontramos en este estudio son las siguientes: es un estudio retrospectivo con todo lo que eso conlleva; se realizó en un único centro, lo que limita la extrapolación de los resultados a otros centros. Como se mencionó anteriormente, únicamente se incluyeron pacientes de bajo riesgo trombótico. Solo tuvimos un paciente al que no se le dio terapia antitrombótica alguna, lo que hace que no podamos sacar conclusiones de este grupo. A pesar de que la clínica de anticoagulantes de este Instituto es de las que cuentan con más experiencia en Latinoamérica, desconocemos el porcentaje de tiempo que los pacientes anticoagulados se encontraban en INR meta. Finalmente, el grupo de pacientes que recibieron doble terapia 
(anticoagulación y ASA) tuvo una cantidad mayor de factores de riesgo cardiovascular, lo que pudo haber influido en las conclusiones (los pacientes únicamente con ASA tuvieron menor riesgo cardiovascular). A pesar de estas debilidades, el número de pacientes así como las variables que se estudiaron nos dan información suficiente para dar paso a un estudio prospectivo al respecto.

\section{Conclusiones}

En este estudio retrospectivo la terapia antitrombótica únicamente con aspirina demostró ser igual de efectiva para prevenir eventos trombóticos que la terapia con AVK y la doble, consistente en aspirina más $\mathrm{AVK}$, para prevenir los eventos de trombosis valvular. Esto da pie a realizar un estudio prospectivo en nuestro Instituto para validar estos hallazgos con el fin de estandarizar la práctica cínica en cuanto a la terapia antitrombótica en pacientes sometidos a cambio valvular aórtico con prótesis biológica y bajo riesgo de trombosis.

\section{Responsabilidades éticas}

Protección de personas y animales. Los autores declaran que para esta investigación no se han realizado experimentos en seres humanos ni en animales.

Confidencialidad de los datos. Los autores declaran que han seguido los protocolos de su centro de trabajo sobre la publicación de datos de pacientes.

Derecho a la privacidad y consentimiento informado. Los autores declaran que en este artículo no aparecen datos de pacientes

\section{Financiación}

Ninguna.

\section{Conflicto de intereses}

Los autores declaran no tener ninguń conflicto de intereses.

\section{Bibliografía}

1. Sun JC, Davidson MJ, Lamy A, et al. Antithrombotic management of patients with prosthetic heart valves: Current evidence an future trends. Lancet. 2009;374:565-76.

2. Van der Wall JC, Umans VA, Schotten J, et al. Antithrombotic strategy after bioprosthetic aortic valve replacement in patients in sinus rhythm: Evaluation of guideline implementation. Eur J Cardiothorac Surg. 2015;8:1-7.

3. Joint Task Force on the Management of Valvular Heart Disease of the European Society of Cardiology (ESC) and the European Association for Cardio-Thoracic SurgeryVahanian A, Alfieri $\mathrm{O}$, Andreotti $\mathrm{F}$, et al. Guidelines on the management of valvular heart disease (version 2012). Eur Heart J. 2012;33: 2451-96.

4. Nishimura R, Otto CM, Bonow RO, et al., American College of Cardiology/American Heart Association Task Force on Practice Guidelines. 2014 AHA/ACC guideline for the management of patients with valvular heart disease: A report of the American College of Cardiology/American Heart Association Task Force on Practice Guidelines. JACC. 2014;63:e57-185.

5. Salem D, Stein PD, Al-Ahmad A, et al. Antithrombotic therapy in valvular heart disease-native and prosthetic: The Seventh ACCP Conference on Antithrombotic and Thrombolytic Therapy. Chest. 2004;126 3 Suppl:82S-457S.

6. British Committee for Standards in Haematology. Guidelines on oral anticoagulation: Third edition. $\mathrm{Br} \mathrm{J}$ Haematol. 1998; 101:374-87.

7. Jamieson W, Cartier PC, Allard M, et al. Surgical management of valvular heart disease. Can J Cardiol. 2004;20 Suppl E:7E-120E.

8. Vaughan P, Waterworth P. Anticoagulation after bioprosthetic aortic valve replacement. J Thorac Cardiovasc Surg. 2006;131:1425.

9. Colli A, Verhoye JP, Leguerrier A, et al. Anticoagulation or antiplatelet therapy of bioprosthetic heart valves recipients: An unresolved issue. Eur J Cardiothorac Surg. 2007;31: 573-7.

10. Brueck M, Kramer W, Vogt P, et al. Antiplatelet therapy early after bioprosthetic aortic valve replacement is unnecessary in patients without thromboembolic risk factors. Eur J Cardiothorac Surg. 2007;32:108-12.

11. Turpie A, Gunstensen J, Hirsh J, et al. Randomised comparison of two intensities of oral anticoagulant therapy after tissue heart valve replacement. Lancet. 1988;1: 1242-5.

12. David T, Ho WI, Christakis GT. Thromboembolism in patients with aortic porcine bioprostheses. Ann Thorac Surg. 1985;40: 229-33.

13. Aramendi J, Agredo J, Llorente A, et al. Prevention of thromboembolism with ticlopidine shortly after valve repair or replacement with a bioprosthesis. J Heart Valve Dis. 1998;7:610-4.

14. Heras M, Chesebro JH, Fuster V, et al. High risk of thromboemboli early after bioprosthetic cardiac valve replacement. J Am Coll Cardiol. 1995;25:1111-9.

15. Orszulak T, Schaff HV, Mullany CJ, et al. Risk of thromboembolism with the aortic Carpentier-Edwards bioprosthesis. Ann Thorac Surg. 1995;59:462-8.

16. Moinuddeen K, Qin J, Shaw R, et al. Anticoagulation is unnecessary after biological aortic valve replacement. Circulation. 1998;10:II95-8.

17. Tiede D, Nishimura RA, Gastineau DA, et al. Modern management of prosthetic valve anticoagulation. Mayo Clin Proc. 1998; 73:665-80.

18. Babin-Ebell J, Schmidt W, Eigel P, et al. Aortic bioprosthesis without early anticoagulation risk of thromboembolism. Thorac Cardiovasc Surg. 1995;43:212-4.

19. Joyce L, Nelson R. Comparison of porcine valve xenografts with mechanical prostheses: A 7 year experience. J Thorac Cardiovasc Surg. 1984;88:102-13.

20. Blair K, Halton AC, White WD, et al. Comparison of anticoagulation regimens after Carpentier-Edwards aortic or mitral valve replacement. Circulation. 1994;90:214-9.

21. Gherli T, Coli A, Fragnito C, et al. Comparing warfarin with aspirin after biological aortic valve replacement: A prospective study. Circulation. 2004;110:496-500.

22. Mistiaen W, van Cauwelaert P, Muylaert P, et al. Thromboembolic events after aortic valve replacement in elderly patients with a Carpentier-Edwards Perimount pericardial bioprosthesis. J Thorac Cardiovasc Surg. 2004;127: 1166-70.

23. Aramendi J, Mestres CA, Martínez-León J, et al. Triflusal versus oral anticoagulation for primary prevention of thromboembolism after bioprosthetic valve replacement (trac): Prospective, randomized, co-operative trial. Eur J Cardiothorac Surg. 2005;27:854-60. 
24. Colli A, Mestres CA, Castella M, et al. Comparing warfarin to aspirin after aortic valve replacement with epic prosthesis: Early results of a prospective randomized trial. Third Biennal Meeting of the Society for Heart Valve Disease, June 17-20 2005. Vancouver BC, Canada [abstract no. P154].

25. Jamieson W, Moffat-Bruce SD, Skarsgard P, et al. Early antithrombotic therapy for aortic valve bioprostheses: Is there an indication for routine use? Ann Thorac Surg. 2007;83: 549-56.

26. Sundt T, Zehar KJ, Dearani JA, et al. Is early anticoagulation with warfarin necessary after bioprosthetic aortic valve replacement? J Thorac Cardiovasc Surg. 2005;129:1024-31. 